\title{
Hepatocellular Carcinomas in Cirrhotic and Noncirrhotic Human Livers Share Angiogenic Characteristics
}

\author{
Wenjiao Zeng, MD, PhD ${ }^{1,5}$, Annette S. H. Gouw, MD, $\mathrm{PhD}^{1}$, Marius C. van den Heuvel, MD, $\mathrm{PhD}^{\mathbf{1}}$, Grietje Molema, \\ $\mathrm{PhD}^{2}$, Sibrand Poppema, MD, $\mathrm{PhD}^{1}$, Eric J. van der Jagt, $\mathrm{MD}, \mathrm{PhD}^{3}$, and Koert $\mathrm{P}$. de Jong, $\mathrm{MD}, \mathrm{PhD}^{4}$ \\ ${ }^{1}$ Department of Pathology and Laboratory Medicine, University Medical Center Groningen, University of Groningen, \\ Groningen, The Netherlands; ${ }^{2}$ Department of Pathology and Laboratory Medicine, Section Medical Biology, University \\ Medical Center Groningen, University of Groningen, Groningen, The Netherlands; ${ }^{3}$ Department of Radiology, University \\ Medical Center Groningen, University of Groningen, Groningen, The Netherlands; ${ }^{4}$ Department of Hepato-Pancreato- \\ Biliary Surgery and Liver Transplantation, University Medical Center Groningen, University of Groningen, Groningen, \\ The Netherlands; ${ }^{5}$ Department of Pathology, Shanghai Medical College, Fudan University, Shanghai, China
}

\begin{abstract}
Background. The antiangiogenic drug sorafenib has been shown to be an effective treatment for hepatocellular carcinoma (HCC) in patients with liver cirrhosis. It might also be effective in noncirrhotic HCC provided that the angiogenic properties of both tumor types are comparable. The aim of this study is to compare endothelial cell dynamics, microvessel density (MVD), and vessel maturation as indirect markers of angiogenesis in human HCC in cirrhotic and noncirrhotic livers.

Materials and Methods. In a tertiary care setting, 70 consecutive HCC tumors were analyzed for endothelial cell dynamics. CD34 was applied to identify tumor microvessels, double immunolabeling Ki67/CD34 and activated caspase-3/CD34 to assess endothelial cell proliferation and apoptosis, and $\alpha$-smooth muscle actin/CD34 for pericyte coverage. These characteristics were compared in cirrhotic $(n=33)$ and noncirrhotic HCCs $(n=37)$. Microvessel density was correlated with radiological signs of hypervascularity as obtained with dynamic four-phase CT scans during the arterial and portal phase of contrast enhancement.
\end{abstract}

Results. Microvessels in cirrhotic and noncirrhotic HCC were mainly mature. In both groups endothelial cell turnover was low and MVD was not different. There was no

(C) The Author(s) 2010. This article is published with open access at Springerlink.com

First Received: 3 August 2009;

Published Online: 20 January 2010

K. P. de Jong, MD, PhD

e-mail: k.p.de.jong@chir.umcg.nl correlation between MVD and venous invasion, tumor size, and turnover of tumor cells or endothelial cells. MVD was negatively correlated with contrast washout in the portal venous phase of $\mathrm{CT}$ scanning. In transplanted patients, MVD was not correlated with survival, whereas in patients after liver resection a high MVD was associated with a better prognosis.

Conclusion. Angiogenic characteristics of HCC in cirrhotic and noncirrhotic livers have a remarkable similarity.

Hepatocellular carcinoma (HCC) generally develops in cirrhotic livers. The various underlying chronic liver diseases that result in cirrhosis have a variable incidence of HCC development. ${ }^{1}$ Viral hepatitis B and C (HBV, HCV) are among the most frequent causes of HCC worldwide. The risk of developing HCC is dependent on the epidemiology of viral hepatitis and alcohol consumption. ${ }^{2}$ Chronic inflammation in the cirrhotic liver induces architectural and functional changes that result in hypoxia, one of the most potent stimuli for angiogenesis. ${ }^{3}$ HCC very rarely develops in patients with normal liver histology (thus without liver cirrhosis) and without underlying viral hepatitis. In contrast to patients with HCC in cirrhotic livers, patients with noncirrhotic HCC often present in advanced stages because they are not participating in screening programs. Additionally, they often do not qualify for liver transplantation because of the large size of the tumors. Recurrence rates of up to $40 \%$ after partial liver resection are reported. ${ }^{4}$

HCC is a highly vascularized tumor and has a predominant arterial supply that can be demonstrated by 
contrast-enhanced computed tomography (CT) scanning or angiography in which hyperattenuation is obtained in the arterial phase and hypoattenuation in the portal phase. ${ }^{5}$ Arterial hypervascularization is one of the diagnostic criteria of HCC in cirrhotic patients. ${ }^{6}$ It is assumed that hypervascularization as seen on imaging techniques correlates with angiogenesis. $^{7}$

Angiogenesis is characterized by destabilization of existing vessels, endothelial cell (EC) proliferation, and vessel sprouting, followed by stabilization of the newly formed vessels. ${ }^{8}$ Because of this, many antiangiogenic treatments target VEGF, which is a crucial growth factor for EC proliferation. ${ }^{9}$ The results of the SHARP trial demonstrate a nearly 3-month survival advantage in those cirrhotic patients with HCC who were treated with sorafenib. ${ }^{10}$ One of the working mechanisms of sorafenib is inhibition of neoangiogenesis by inhibition of the vascular endothelial growth factor receptors (VEGFR). ${ }^{11}$ It is unknown whether $\mathrm{HCC}$ in noncirrhotic liver has similar angiogenic aspects as HCC in cirrhotic livers. Similarities between the angiogenic characteristics of HCC in cirrhotic and noncirrhotic livers could lend support to sorafenib treatment for advanced HCC in noncirrhotic patients.

The aim of the present study was (1) to compare the angiogenic status of HCC in cirrhotic and noncirrhotic livers and (2) to correlate MVD with tumor-, patient-, and radiological characteristics of HCC. To this end, we analyzed the dynamics of ECs in the tumor vessels in HCC by the quantification of MVD, by the apoptotic and proliferation rate of ECs in tumor microvessels, and by the tumor vessel's pericyte coverage, which is considered to be a sign of mature vessels.

\section{PATIENTS AND METHODS}

\section{Patients}

A total of 70 anonymized HCC tumor specimens from 69 consecutive patients were analyzed according to recent national guidelines. In 31 cases, HCC developed in cirrhotic livers, and these patients underwent a liver transplantation $(n=30)$ or a local resection $(n=1)$. The causes of the cirrhosis were $\operatorname{HBV}(n=8)$ or $\operatorname{HCV}(n=9)$, cryptogenic cirrhosis $(n=5)$, primary biliary cirrhosis, alcohol, autoimmune hepatitis, $\alpha 1$-antitrypsin deficiency ( $n=2$ each), and biliary atresia $(n=1)$. In two additional patients, each with a cirrhotic liver, biopsies of the HCC were available for analysis. In the 30 liver explants, solitary tumor nodules were found in 13 cases, 2 nodules were found in 8 cases, 3 nodules in 3 cases, and the remaining 6 cases had more than 3 nodules. A representative sample of 1 nodule per case was obtained for the analysis. Another 35 cases developed HCC in normal, noncirrhotic, nonfibrotic livers, and in none of these patients were $\mathrm{HBV}$ or $\mathrm{HCV}$ antibodies detectable. All these patients underwent a partial liver resection. In 1 patient, a recurrent $\mathrm{HCC}$ in a noncirrhotic liver was resected 16 months after a previous local resection, and both tumors were included in the study. The follow-up of this patient was calculated from her first operation. A tumor biopsy of another noncirrhotic patient was added for analysis. In the noncirrhotic group, 29 patients had single tumor nodules. The remaining 6 patients had more than 1 tumor nodule, and in those cases a representative sample of 1 tumor nodule per case was obtained for the analysis. None of the patients had undergone any previous treatment for HCC. Of the 35 noncirrhotic patients, 5 were excluded from the survival analysis because of early (within 3 months) postoperative mortality $(n=3)$ or because of noncurative resections $(n=2)$. Of the 30 transplanted patients, 2 were excluded because of early postoperative mortality. The follow-up for the patients consisted of $\alpha$-fetoprotein serum levels, thoracic $\mathrm{X}$-rays, and ultrasound examinations of the liver remnant every 3 months during the first 2 years after partial liver resection and every 6 months thereafter, until 5 years after the resection date. In case of suspicious findings, CT scans of the thorax and abdomen were performed in order to detect resectable recurrences. In cases of unresectable recurrences, patients were treated with best supportive care. In patients who had liver transplant, routine follow-up was done according to our protocol, which included ultrasound of the liver, $\alpha$-fetoprotein serum levels, and thoracic X-rays every 6 months.

\section{Immunohistochemistry}

Immunohistochemical staining with CD34 (clone Qbend 10, ready-to-use dilution, Immunotech, Marseille, France), Ki 67 (DAKO, Glostrup, Denmark. 1:75), and anticleaved caspase-3 (Cell Signaling Technology, Danvers, MA, 1:50) was used on $4 \mu \mathrm{m}$ paraffin sections. Single immunostaining with anti-CD34 was performed to detect MVD in the tumors. Double immunostaining of anti-CD34 and anticleaved caspase- 3 were performed as described previously. ${ }^{12}$ Double immunostaining using anti-CD34 and antiKi67 was performed after microwave antigen retrieval. Sections were incubated with anti-Ki67 followed by RAMPO/GARPO and DAB to develop the staining reaction. After incubation with anti-CD34, GAMAP and Fast Red were used for the staining reaction and hematoxylin for nuclear counterstaining. Double immunostaining of CD34/ $\alpha$ SMA was performed to detect vascular pericyte coverage in a method similar to the CD34/Ki67 double labeling but without any prior antigen retrieval. 


\section{Evaluation of Immunohistochemical Staining}

Grading of HCC was done according to Edmondson and Steiner and the growth patterns were identified as trabecular, acinar, solid, and scirrhous. The Chalkley point overlap morphometric technique was used for quantification of the MVD at three "hot spots" on the CD34-stained sections, as described previously. ${ }^{12,13}$ First, tumors were scanned at low magnification to identify areas with high MVD ("hot spots"). Then, at $\times 200$ magnification, an eyepiece graticule containing 25 randomly positioned dots was rotated to reach the maximum number of points overlapping with a $\mathrm{CD} 34$ positive structure, that is, the vessels in the vascular "hot spot." The number of overlaying dots was counted. $\alpha$ SMA coverage of vessels was calculated as the number of dots within or on the $\alpha$-SMApositive vessels as a percentage of the total number of dots overlaying CD34-positive structures. On CD34/Ki67 double-stained sections, Ki67 labeling was determined in a $\times 400$ microscopic field, at the same 3 "hot spots" as used for MVD counting. The vascular Ki67 labeling index (LI) was defined as the number of Ki67 +/CD34 + EC nuclei per total number of ECs. The Ki67 LI of tumor cells was obtained by counting the number of Ki67 positively stained tumor cells per 300 individual tumor cells in each area. In CD34/activated caspase-3 double-labeled sections, the activated caspase- 3 positive HCC cells and activated caspase- 3 positive ECs were calculated in three "hot spots" at $400 \times$ magnification. For each case, the apoptotic index (AI) was calculated as the number of apoptotic cells $x$ $100 /$ number of high-power fields.

\section{Analysis of CT Images}

Images were analyzed using the change in attenuation of dynamic four-phase CT scanning during the arterial and portal phase of contrast enhancement. Only the source data of CT scans performed after 2002 were available for analysis. The enhancement (in Houncefield units; HU) in the tumor and the surrounding parenchyma was recorded. In tumors with a heterogeneous enhancement, the most hyperattenuating region was identified in order to obtain the most reliable comparison with MVD, which was scored in the vascular "hot spot." The relative enhancement of the tumor was compared with that of the surrounding parenchyma in the arterial and portal phases according to the equation:

Relative enhancement $=\frac{\mathrm{HU} \text { tumor }-\mathrm{HU} \text { parenchyma }}{\mathrm{HU} \text { parenchyma }}$

The relative enhancement was correlated with MVD. We chose for calculation of the relative enhancement to eliminate interindividual differences in the contrast medium enhancement in HCC tumor nodules versus the adjacent parenchyma. This difference is highly dependent on various factors including patients' physiology, for example, the cardiac output in the hyperdynamic circulation of cirrhotic patients. ${ }^{14}$

\section{Statistics}

The results of the vascular dynamics in cirrhotic versus noncirrhotic HCCs were presented as medians with interquartile ranges (IQR) and were compared using the MannWhitney test. MVD in relation to tumor grade was analyzed using ANOVA. Categorical variables were analyzed using the chi-square test or the Fisher exact test, where appropriate. Survival analysis was performed using the Kaplan-Meier method, with the log-rank test for comparison. Median survival and 95\% confidence intervals (95\% CI) were given together with 5-year survival data. Pearson correlation coefficients were presented for data with a normal distribution. Variables with a nonparametric distribution were $\log$ transformed in order to normalize the data.

\section{RESULTS}

\section{EC Apoptosis and Proliferation}

EC apoptosis and proliferation are rare events in tumor microvessels. In 31 of the 37 noncirrhotic HCCs and 29 of the 33 cirrhotic HCCs, no apoptotic ECs were identified. In the remaining tumors, apoptotic ECs could rarely be identified (Fig. 1). EC proliferation was also rare in both types of HCCs (Fig. 2). Nevertheless, the median number (IQR) per high-power field in noncirrhotic HCCs was higher than in cirrhotic HCCs (Table $1, P=.011$ ).

\section{Similar MVD in HCCs in Cirrhotic versus Noncirrhotic Livers}

CD34 positive tumor vessels demonstrated a rather uniform pattern of staining. A striking change in CD34 expression was visible at the interface of an HCC and adjacent parenchyma (Fig. 3). In the parenchyma, sinusoids were faintly stained with a progressive increase in staining intensity toward the tumor. Of note is the change in morphology, with the sinusoids in the normal liver demonstrating obvious lumina, whereas in the tumor itself the vessel lumen was often slitlike and the vessels were tortuous when a sinusoidal-like pattern was present. Quantification of MVD revealed no difference between noncirrhotic and cirrhotic HCCs (Table 1). 


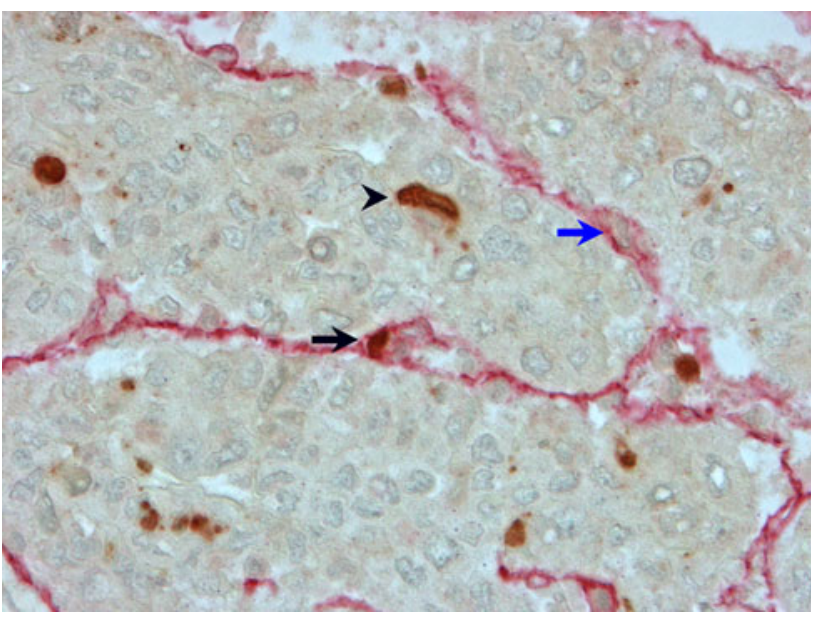

FIG. 1 Immunohistochemistry of hepatocellular carcinoma demonstrating apoptosis in tumor microvessels. Apoptotic EC (black arrow) within the tumor microvessel. Double immunolabeling: anti-CD34 (red), anti-activated caspase 3 (brown). Negative EC nucleus: blue arrow. Apoptotic tumor cell: black arrowhead

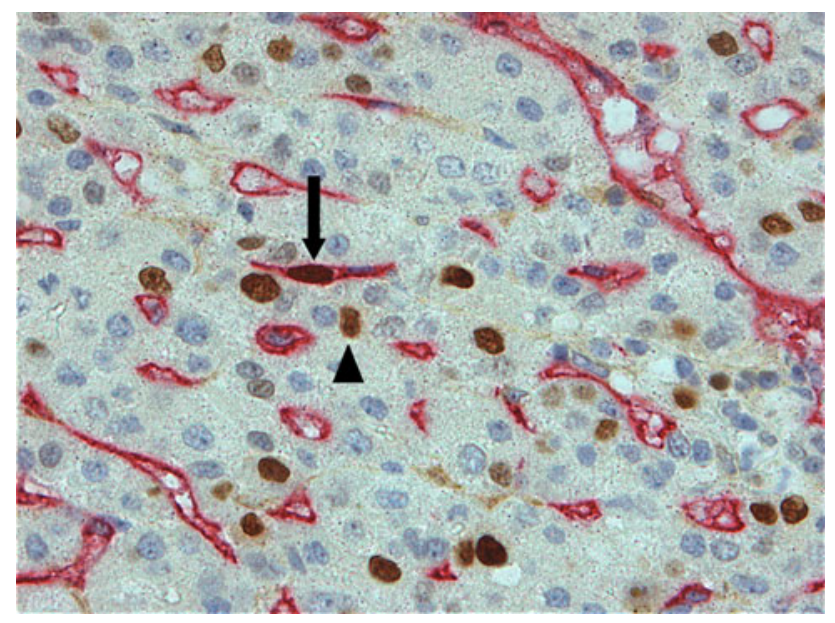

FIG. 2 Immunohistochemistry of hepatocellular carcinoma demonstrating proliferation in tumor microvessels. Double immunolabeling: anti-CD34 (red), Ki67 (brown). Proliferating ECs (arrow) and proliferating tumor cells (arrow head) are clearly visible

\section{MVD in Relation to Vascular Invasion}

Because vascular invasion is an important adverse prognostic variable, MVD was compared in tumors with vascular invasion versus those without vascular invasion in cirrhotic and noncirrhotic livers separately. The mean MVD in cirrhotic HCCs was comparable in tumors with vascular invasion versus those without vascular invasion (Table 1). In noncirrhotic livers, tumors without vascular invasion tended to have a lower MVD compared with tumors with vascular invasion $(P=.07$, Table 1$)$.

\section{aSMA-Positive Cells}

The majority of tumor microvessels are covered by $\alpha$ SMA-positive cells. Microvessels that are double positive for CD34 and $\alpha$ SMA were regarded to be mature vessels. The median percentage (IQR) of CD34 +/ $\alpha \mathrm{SMA}+$ vessels in noncirrhotic and cirrhotic HCCs was comparable (Table 1). These numbers suggest that the majority of HCC microvessels were stabilized, quiescent vessels.

\section{Proliferation and Apoptosis in Tumor Cells}

The proliferation rate of tumor cells in noncirrhotic versus cirrhotic HCCs was no different (Table 1). The rate of apoptotic tumor cells in general was low, despite the fact that they could be easily identified if present (Fig. 1). The median number of apoptotic tumor cells per high-power field in noncirrhotic livers was slightly different from that in cirrhotic livers (Table 1).

\section{MVD in Relation to Tumor and EC Characteristics}

Of the $70 \mathrm{HCCs}, 6$ were graded as (Edmonson Steiner) grade 1,39 as grade 2 , and 25 as grade 3 . ANOVA with post hoc comparisons revealed a significant $(P<.05)$ difference between MVD in grade 1 (mean 9.4, standard deviation [SD] 2.8) versus in grade 2 (mean 13.1, SD 3.4) HCCs.

MVD was not correlated with tumor size, with the rate of tumor cell proliferation or apoptosis, or with the rate of EC proliferation or apoptosis in tumor microvessels. In addition, no correlation was encountered between MVD and the number of $\alpha$ SMA-positive CD34-positive microvessels (mature vessels).

\section{MVD and Contrast Washout}

MVD is negatively correlated with contrast washout but is not correlated with contrast supply. In general, the tumors were hyperintense in the arterial phase; the mean number of $\mathrm{HU}$ in the tumor was $17 \%$ (range $-56 \%$ to $+57 \%$ ) higher compared with the surrounding parenchyma. No correlation $(r=-.23, P=.52)$ was found between MVD and the relative contrast enhancement of the tumor during the arterial phase. In the portal phase, most of the tumors were hypointense; the mean number of $\mathrm{HU}$ was $15 \%$ (range $-35 \%$ to $+19 \%$ ) lower than the surrounding parenchyma. A negative correlation $(r=-.75, P=.012)$ was found between MVD and the enhancement of the tumor in the portal phase. Thus, the higher the MVD in the tumors the less contrast was present in the tumor during the portal phase of contrast enhancement. 
TABLE 1 Clinicopathological, angiogenic and survival characteristics in patients with HCC in noncirrhotic versus cirrhotic livers

\begin{tabular}{llll}
\hline & Noncirrhotic HCC & Cirrhotic HCC & $P$ value \\
\hline Gender (male:female) & $16: 14$ & $25: 3$ & .001 \\
Mean age (range) & $58.9(20-83)$ & $50.2(2-68)$ & .06 \\
No. of nodules $1 / 2-3 />3$ & $26 / 2 / 2$ & $8 / 10 / 10$ & $<.0001$ \\
Size of largest tumor median (range) & $13.0(3.0-25.0)$ & $2.7(1.0-6.0)$ & $<.0001$ \\
$\alpha$ fetoprotein serum level median (range) & $6.8(3-93,933)$ & $21.5(2-16,000)$ & $>.6$ \\
Endothelial cell proliferation & $0.03(0.04)$ & $0.02(0.02)$ & .011 \\
MVD & $13.0(6.0)$ & $11.3(3.0)$ & .46 \\
MVD, mean (range) & & & .07 \\
Venous invasion & $13.3(6-22)$ & & .9 \\
No venous invasion & $10.9(6-16)$ & & .27 \\
Venous invasion & & $11.9(8-19)$ & .23 \\
No venous invasion & & $63.0(4-19)$ & .09 \\
$\%$ Mature vessels & $79.0(61.0)$ & $5.0(8.4)$ & .036 \\
$\%$ Tumor cell proliferation & $5.8(15.1)$ & $0.0(0.67)$ & .05 \\
Apoptotic tumor cells & $0.33(1.33)$ & $112(60-165)$ & \\
Median survival, months (95\% CI) & $38(11-65)$ & & \\
Low MVD & $12.2(5.4-19.0)$ & Not reached & \\
High MVD & $58(24.8-90.7)$ & & \\
Low MVD & & & \\
High MVD & & & \\
\hline
\end{tabular}

values (interquartile range) unless stated otherwise

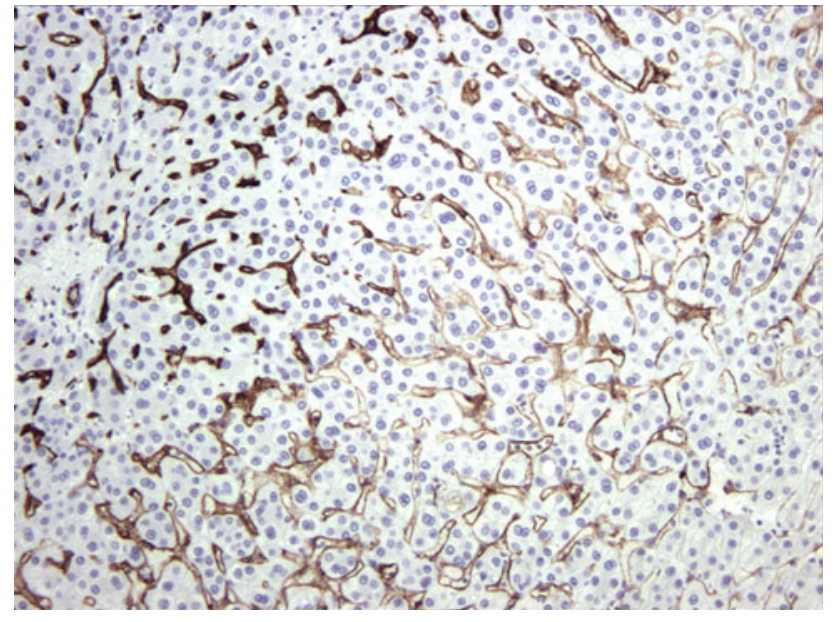

FIG. 3 Immunolabeling with CD34 (brown), staining the border of a hepatocellular carcinoma with adjacent nontumorous liver parenchyma. The adjacent liver parenchyma (lower right-hand corner) shows a very faint staining with a gradual increase in staining intensity toward the tumor (upper left border)

\section{MVD and Patient Survival}

First, we analyzed survival in cirrhotic and noncirrhotic patients separately because of the major differences in the patient and disease characteristics found in the 2 groups (Table 1). Median survival in transplanted patients (112 months) was better than in resected patients (38 months, $P=.036$ ). The 5 -year survival rates were $66.8 \%$ in transplanted patients and $44.3 \%$ in resected patients. In order to analyze the impact of MVD on survival, we categorized MVD into "low" ( $<$ median MVD value) and "high" values ( $>$ median MVD value). Survival of patients with high-MVD tumors was compared with that of patients with low-MVD tumors separately for transplanted and resected patients. Survival in transplanted patients was no different in the low versus the high MVD values (Fig. 4; Table 1). In contrast, in the resection group, the median survival in patients with tumors with a high MVD (58 months) was longer $(P=.05)$ compared with the median survival in patients with low-MVD tumors (12.2 months, Fig. 4; Table 1).

\section{DISCUSSION}

In this study we analyzed the angiogenic characteristics of HCC in cirrhotic versus noncirrhotic livers at three different levels: quantification of proliferation and apoptosis of ECs in tumor vessels at the cellular level, quantification of MVD at the tumor-tissue level, and analysis of tumor vascularization as seen by contrastenhanced CT imaging. The main conclusion is that the angiogenic characteristics of $\mathrm{HCC}$ in cirrhotic livers are to a large extent similar to those in noncirrhotic livers. Additionally, microvessels in both tumor types exhibit a very low turnover rate and mainly have the characteristics of stable mature vessels. Indirect evidence of angiogenic activity could be provided by EC dynamics, for example, proliferative activity and apoptosis. Our findings of a low 


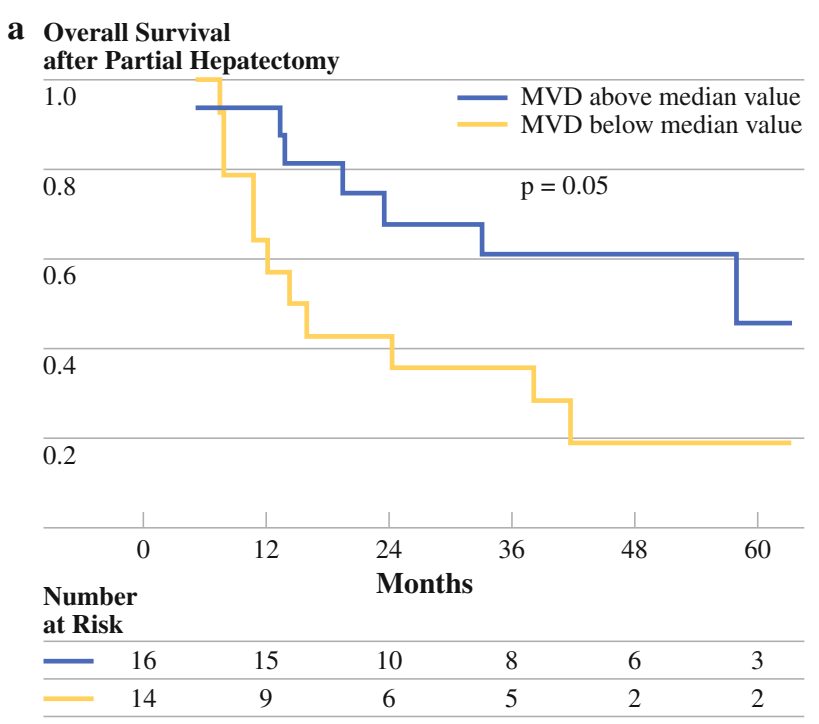

b Overall Survival

after Liver Transplant

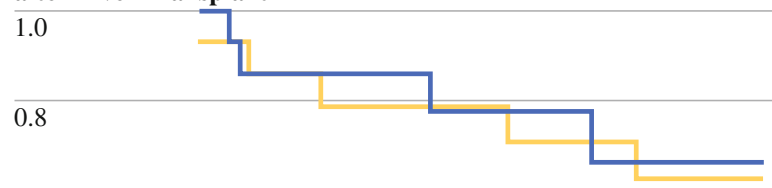

$\overline{0.6}$
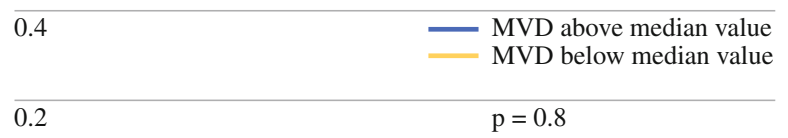

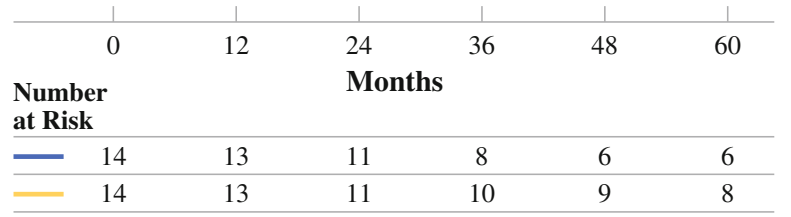

FIG. 4 Overall survival of patients with HCC in noncirrhotic livers after partial hepatectomy (left panel) or patients with HCC in cirrhotic livers after liver transplantation (right panel), stratified according to microvessel density (MVD). MVD is divided into values above and below the median value

proliferative and low apoptotic rate in ECs, the presence of a high $\alpha$ SMA coverage rate, and the absence of a positive correlation between hypervascularity on imaging and tumor MVD are compatible with a rather stable EC compartment in tumor vessels in HCCs, irrespective of the presence of cirrhosis. These results are in agreement with the findings of our previous study in which we quantified gene and protein expression of VEGF-A, its receptors VEGFR-1 and VEGFR-2, and the angiopoietin/Tie 2 system in HCC of cirrhotic and noncirrhotic patients. ${ }^{15} \mathrm{We}$ found no increase in VEGF-A levels, nor the characteristic pattern of increased angiopoietin-2/Tie-2 concomitant with decrease of angiopoietin-1, which is the usual profile for tumor angiogenesis as seen in highly angiogenic tumors, for example, renal cell carcinoma. ${ }^{15}$

Although angiogenic sprouting, that is, new capillary bud formation from preexisting vessels, was initially thought to be the main mechanism of new vessel formation, various other mechanisms for vascularization of tumors were put forward. ${ }^{16}$ In the extremely rich vascularized liver, especially, the process of co-option of preexistent vessels-sinusoids-might be a suitable option. ${ }^{17}$ Co-option is a process in which the tumor hijacks (co-opts) preexistent vessels of the host tissue in order to obtain vascularization for ongoing tumor growth and has been demonstrated in metastatic brain tumors in mice. ${ }^{18}$

A progressive increase in CD34 expression by hepatic sinusoidal ECs parallels the progression from low-grade to high-grade dysplastic nodules into frank HCC. ${ }^{19}$ This increased expression is considered to be a sign of angiogenesis. ${ }^{7,20-23}$ Another possibility, however, is that changes in the environment (from normal hepatocytes to malignant and premalignant cells) induce an altered phenotype of ECs; increasing CD34 expression on ECs might represent a bystander effect of changing influences from adjacent cells or growth factors.

An analysis of the literature on the relationship between MVD and survival is presented in Table 2, which mainly presents data on patients with resected HCCs. It can be concluded from this table that CD34 is the most widely used antibody to identify microvessels and that the association between MVD in tumor microvessels and prognosis is highly variable. Most reports have found that a high MVD is associated with a poor prognosis; others found no association and two (including ours) found a favorable outcome in resected patients with high-MVD tumors. These differences can probably be explained by differences in the underlying causes of the liver disease in the reported studies. In addition, conflicting results have been published on the correlation between hypervascularity on contrastenhanced CT and MVD in HCC. In one study, a correlation between MVD and arterial and portal phase contrast enhancement was found, whereas others found no correlation. ${ }^{24-26}$ In the present study, we found no correlation between MVD and contrast enhancement in the tumor in the arterial phase and a negative correlation in the portal phase. These findings suggest that a high MVD is associated more with rapid washout of contrast than with a large arterial blood supply.

We found a remarkable difference in the relationship of MVD to survival; no effect of MVD was found in patients after liver transplantation, whereas patients with a high MVD had a better prognosis after partial liver resection than those with a low MVD. One possible explanation for the fact that MVD does not relate to survival in transplanted patients could be that metastases most frequently 
TABLE 2 Summary of published series on MVD and prognosis of HCC after resection or liver transplantation

\begin{tabular}{|c|c|c|c|c|c|c|}
\hline $\begin{array}{l}\text { Year of publication } \\
\text { and Author }\end{array}$ & $\begin{array}{l}\text { Number } \\
\text { of patients }\end{array}$ & Therapy $^{\mathrm{a}}$ & $\begin{array}{l}\text { Microvessel } \\
\text { staining }^{\mathrm{b}}\end{array}$ & $\begin{array}{l}\text { Prognosis of patients } \\
\text { with high } \mathrm{MVD}^{\mathrm{c}}\end{array}$ & $\begin{array}{l}\text { Correlation of } \\
\text { hypervascularity } \\
\text { and } \mathrm{MVD}^{\mathrm{d}}\end{array}$ & $\begin{array}{l}\text { Correlation of high } \\
\text { VEGF and } \text { MVD }^{\mathrm{e}}\end{array}$ \\
\hline 1997 Tanigawa $^{29}$ & 43 & RES & $\begin{array}{l}\text { CD34 } \\
\text { vWF }\end{array}$ & Poor (DFS/OS) & NE & $\mathrm{NE}$ \\
\hline $1998 \mathrm{El} \mathrm{Assal}^{25}$ & 71 & RES & $\mathrm{vWF}$ & Poor (DFS) & 0 & 0 \\
\hline $1999 \operatorname{Sun}^{30}$ & 78 & RES & CD34 & Poor (DFS) & NE & NE \\
\hline 2002 Poon $^{21}$ & 100 & RES & $\begin{array}{l}\text { CD34 } \\
\text { vWF }\end{array}$ & $\begin{array}{l}\text { Poor (DFS) } \\
\text { Poor (DFS) }\end{array}$ & $\mathrm{NE}$ & NE \\
\hline 2004 Nanashima $^{31}$ & 81 & RES & CD34 & Favorable (DFS/OS) & NE & NE \\
\hline $2005 \mathrm{Ho}^{32}$ & 86 & RES & $\begin{array}{l}\text { CD34 } \\
\text { CD105 }\end{array}$ & $\begin{array}{l}\text { Comparable (DFS) } \\
\text { Comparable (DFS) }\end{array}$ & NE & $\mathrm{NE}$ \\
\hline 2006 Yang $^{33}$ & 113 & RES & $\begin{array}{l}\text { CD34 } \\
\text { CD105 }\end{array}$ & $\begin{array}{l}\text { Comparable (OS) } \\
\text { Poor (OS) }\end{array}$ & $\mathrm{NE}$ & + \\
\hline 2006 Zhang $^{34}$ & 82 & LT & CD34 & Poor (DFS, univariate) & NE & + \\
\hline $2007 \mathrm{Yao}^{35}$ & 105 & RES & $\begin{array}{l}\text { CD34 } \\
\text { CD105 }\end{array}$ & $\begin{array}{l}\text { Poor (DFS/OS) } \\
\text { Poor (DFS/OS) }\end{array}$ & NE & $\begin{array}{l}0 \\
+\end{array}$ \\
\hline Present series & $\begin{array}{l}32 \\
30\end{array}$ & $\begin{array}{l}\text { RES } \\
\text { LT }\end{array}$ & $\begin{array}{l}\text { CD34 } \\
\text { CD34 }\end{array}$ & $\begin{array}{l}\text { Favorable (DFS/OS) } \\
\text { Comparable (DFS/OS) }\end{array}$ & 0 (art) $-($ portal $)$ & $\mathrm{NE}$ \\
\hline
\end{tabular}

$N E$ not evaluated

a Type of treatment for HCC. RES partial liver resection, $L T$ liver transplantation

b Antibody used for detection of microvessels. $v W F$ anti-von Willebrand factor (factor VIII), CD34 anti-CD34 antibody, CD105 anti-CD105 antibody

${ }^{c}$ Prognosis of patients with tumors with high MVD as compared to those with low MVD. DFS disease-free survival, OS overall survival

${ }^{\mathrm{d}}$ Correlation of hypervascularity as seen on contrast enhanced CT or angiography with MVD. +/-/0, positive, negative or no correlation

${ }^{\mathrm{e}}$ Correlation of vascular endothelial growth factor (VEGF) with MVD. +/-/0, positive, negative, or no correlation

occur in the liver first. Thus, independent of a high or low metastatic propensity, the intrahepatic metastases are removed during liver transplantation, whereas following partial liver resection the remnant liver can harbor clinically undetectable metastases that give rise to recurrences in due time. Our finding that patients with HCC with a low MVD have a worse prognosis after partial liver resection might be explained by the well-established phenomenon of a more aggressive behavior of hypoxic tumor cells. ${ }^{27,28}$ Based on this, low-MVD HCCs might have a higher fraction of hypoxic tumor cells resulting in a more aggressive tumor cell population, with a greater chance of intrahepatic metastases.

There are three main limitations to this study: first, its retrospective nature; second, the limited number of patients, which could result in a type II error; and, third, the fact that we analyzed clinically detectable-advancedHCCs and not early HCCs in which the angiogenic dynamics might be different. However, the low incidence of HCC in noncirrhotic livers hampers the acquisition of large numbers of patients in a reasonable time span. In addition, the intervention of angiogenesis using sorafenib is only indicated in advanced HCC and, as such, the angiogenic balance is relevant when examining advanced HCC.
In conclusion, we found a low EC proliferation rate, a high $\alpha$ SMA coverage of vessels, and no correlation between the arterial blood supply and MVD both in cirrhotic and noncirrhotic HCCs. It appears that the EC compartment of HCC microvessels demonstrates a rather low turnover rate and is comparable in cirrhotic and noncirrhotic HCC. Until now the effect of sorafenib has only been proven in patients with advanced HCC, with a background of liver cirrhosis. Given the rarity of HCC in noncirrhotic liver, it will be difficult to prove the efficacy of sorafenib in patients with noncirrhotic HCC in a randomized trial. However, the results of the present study suggest a similarity in angiogenic status of HCC in cirrhotic and noncirrhotic livers and, as such, support the use of sorafenib in advanced HCC in noncirrhotic patients as well.

ACKNOWLEDGMENT Part of this study was supported by the Bernouilli Scholarship fund of UMCG. This funding was independent of the work.

OPEN ACCESS This article is distributed under the terms of the Creative Commons Attribution Noncommercial License which permits any noncommercial use, distribution, and reproduction in any medium, provided the original author(s) and source are credited. 


\section{REFERENCES}

1. El Serag HB, Rudolph KL. Hepatocellular carcinoma: epidemiology and molecular carcinogenesis. Gastroenterology. 2007;132: 2557-76.

2. Ribes J, Cleries R, Esteban L, Moreno V, Bosch FX. The influence of alcohol consumption and hepatitis $\mathrm{B}$ and $\mathrm{C}$ infections on the risk of liver cancer in Europe. J Hepatol. 2008;49:233-42.

3. Medina J, Arroyo AG, Sanchez-Madrid F, Moreno-Otero R. Angiogenesis in chronic inflammatory liver disease. Hepatology. 2004;39:1185-95.

4. Lubrano J, Huet E, Tsilividis B, François A, Goria O, Riachi G, et al. Long-term outcome of liver resection for hepatocellular carcinoma in noncirrhotic nonfibrotic liver with no viral hepatitis or alcohol abuse. World J Surg. 2008;32:104-9.

5. Pandharipande PV, Krinsky GA, Rusinek H, Lee VS. Perfusion imaging of the liver: current challenges and future goals. Radiology. 2005;234:661-73.

6. Bruix J, Sherman M, Llovet JM, Beaugrand M, Lencioni R, Burroughs $\mathrm{AK}$, et al. Clinical management of hepatocellular carcinoma. Conclusions of the Barcelona-2000 EASL conference. European Association for the Study of the Liver. J Hepatol. 2001;35:421-30.

7. Semela D, Dufour JF. Angiogenesis and hepatocellular carcinoma. J Hepatol. 2004;41:864-80.

8. Carmeliet P. Angiogenesis in life, disease and medicine. Nature. 2005;438:932-6.

9. Siemann DW, Bibby MC, Dark GG, Dicker AP, Eskens FA, Horsman MR, et al. Differentiation and definition of vasculartargeted therapies. Clin Cancer Res. 2005;11:416-20.

10. Llovet JM, Ricci S, Mazzaferro V, Hilgard P, Gane E, Blanc JF, et al. Sorafenib in advanced hepatocellular carcinoma. $N$ Engl $J$ Med. 2008;359:378-90.

11. Wilhelm SM, Carter C, Tang L, Wilkie D, McNabola A, Rong H, et al. BAY 43-9006 exhibits broad spectrum oral antitumor activity and targets the RAF/MEK/ERK pathway and receptor tyrosine kinases involved in tumor progression and angiogenesis. Cancer Res. 2004;64:7099-109.

12. de Jong KP, Vermeulen. PB, Van Marck E, Boot M, Gouw ASH. Endothelial cell apoptosis in the context of quantification of angiogenesis in solid human adenocarcinomas: a novel double immunolabelling technique to identify endothelial cell apoptosis. Eur J Cancer. 2006;42:97-100.

13. Vermeulen PB, Gasparini G, Fox SB, Colpaert C, Marson LP, Gion M, et al. Second international consensus on the methodology and criteria of evaluation of angiogenesis quantification in solid human tumours. Eur J Cancer. 2002;38:1564-79.

14. Bae KT, Heiken JP. Scan and contrast administration principles of MDCT. Eur Radiol. 2005;15 Suppl 5:E46-E59.

15. Zeng W, Gouw AS, van den Heuvel MC, Zwiers PJ, Zondervan $\mathrm{PE}$, Poppema $\mathrm{S}$, et al. The angiogenic makeup of human hepatocellular carcinoma does not favor vascular endothelial growth factor/angiopoietin-driven sprouting neovascularization. Hepatology. 2008;48:1517-27.

16. Dome B, Hendrix MJ, Paku S, Tovari J, Timar J. Alternative vascularization mechanisms in cancer: pathology and therapeutic implications. Am J Pathol. 2007;170:1-15.

17. Ellis LM, Hicklin DJ. VEGF-targeted therapy: mechanisms of anti-tumour activity. Nat Rev Cancer. 2008;8:579-91.

18. Leenders WP, Kusters B, Verrijp K, Maass C, Wesseling P, Heerschap A, et al. Antiangiogenic therapy of cerebral melanoma metastases results in sustained tumor progression via vessel cooption. Clin Cancer Res. 2004;10:6222-30.

19. Park YN, Yang CP, Fernandez GJ, Cubukcu O, Thung SN, Theise ND. Neoangiogenesis and sinusoidal "capillarization" in dysplastic nodules of the liver. Am J Surg Pathol. 1998;22: 656-62.

20. Roncalli M, Roz E, Coggi G, Di Rocco MG, Bossi P, Minola E, et al. The vascular profile of regenerative and dysplastic nodules of the cirrhotic liver: implications for diagnosis and classification. Hepatology. 1999;30:1174-8.

21. Poon RT, Ng IO, Lau C, Yu WC, Yang ZF, Fan ST, et al. Tumor microvessel density as a predictor of recurrence after resection of hepatocellular carcinoma: a prospective study. J Clin Oncol. 2002;20:1775-85.

22. Mitsuhashi N, Shimizu H, Ohtsuka M, Wakabayashi Y, Ito H, Kimura F, et al. Angiopoietins and Tie-2 expression in angiogenesis and proliferation of human hepatocellular carcinoma. Hepatology. 2003;37:1105-13.

23. Qin LX, Tang ZY. Recent progress in predictive biomarkers for metastatic recurrence of human hepatocellular carcinoma: a review of the literature. J Cancer Res Clin Oncol. 2004;130: 497-513.

24. Wang B, Gao ZQ, Yan X. Correlative study of angiogenesis and dynamic contrast-enhanced magnetic resonance imaging features of hepatocellular carcinoma. Acta Radiol. 2005;46:353-8.

25. El-Assal ON, Yamanoi A, Soda Y, Yamaguchi M, Igarashi M, Yamamoto A, et al. Clinical significance of microvessel density and vascular endothelial growth factor expression in hepatocellular carcinoma and surrounding liver: possible involvement of vascular endothelial growth factor in the angiogenesis of cirrhotic liver. Hepatology. 1998;27:1554-62.

26. Kim CK, Lim JH, Park CK, Choi D, Lim HK, Lee WJ. Neoangiogenesis and sinusoidal capillarization in hepatocellular carcinoma: correlation between dynamic CT and density of tumor microvessels. Radiology. 2005;237:529-34.

27. Sullivan R, Graham CH. Hypoxia-driven selection of the metastatic phenotype. Cancer Metastasis Rev. 2007;26:319-31.

28. Tatum JL, Kelloff GJ, Gillies RJ, Arbeit JM, Brown JM, Chao $\mathrm{KS}$, et al. Hypoxia: importance in tumor biology, noninvasive measurement by imaging, and value of its measurement in the management of cancer therapy. Int $J$ Radiat Biol.2006;82: 699-757.

29. Tanigawa N, Lu C, Mitsui T, Miura S. Quantitation of sinusoidlike vessels in hepatocellular carcinoma: its clinical and prognostic significance. Hepatology. 1997;26:1216-23.

30. Sun HC, Tang ZY, Li XM, Zhou YN, Sun BR, Ma ZC. Microvessel density of hepatocellular carcinoma: its relationship with prognosis. J Cancer Res Clin Oncol. 1999;125:419-26.

31. Nanashima A, Yano H, Yamaguchi H, Tanaka K, Shibasaki S, Sumida Y, et al. Immunohistochemical analysis of tumor biological factors in hepatocellular carcinoma: relationship to clinicopathological factors and prognosis after hepatic resection. J Gastroenterol. 2004;39:148-54.

32. Ho JW, Poon RT, Sun CK, Xue WC, Fan ST. Clinicopathological and prognostic implications of endoglin (CD105) expression in hepatocellular carcinoma and its adjacent non-tumorous liver. World J Gastroenterol. 2005;11:176-81.

33. Yang LY, Lu WQ, Huang GW, Wang W. Correlation between CD105 expression and postoperative recurrence and metastasis of hepatocellular carcinoma. BMC Cancer. 2006;6:110.

34. Zhang Q, Chen X, Zhou J, Zhang L, Zhao Q, Chen G, et al. CD147, MMP-2, MMP-9 and MVD-CD34 are significant predictors of recurrence after liver transplantation in hepatocellular carcinoma patients. Cancer Biol Ther. 2006;5:808-14.

35. Yao Y, Pan Y, Chen J, Sun X, Qiu Y, Ding Y. Endoglin (CD105) expression in angiogenesis of primary hepatocellular carcinomas: analysis using tissue microarrays and comparisons with CD34 and VEGF. Ann Clin Lab Sci. 2007;37:39-48. 